\title{
Partial Integrability Conditions and an Integrating Algorithm for Fully Rheonomous Affine Constraints
}

\author{
Tatsuya Kai \\ Department of Applied Electronics, Faculty of Industrial Science and Technology, Tokyo University of Science, \\ Tokyo, Japan \\ Email: kai@rs.tus.ac.jp
}

Received 4 May 2014; revised 4 June 2014; accepted 11 June 2014

Copyright (@ 2014 by author and Scientific Research Publishing Inc.

This work is licensed under the Creative Commons Attribution International License (CC BY). http://creativecommons.org/licenses/by/4.0/

(c) (i) Open Access

\begin{abstract}
In this paper, integrability conditions and an integrating algorithm of fully rheonomous affine constraints (FRACs) for the partially integrable case are studied. First, some preliminaries on the FRACs are illustrated. Next, necessary and sufficient conditions on the partially integrable case for the FRACs are derived. Then, an integrating algorithm to calculate independent first integrals of the FRACs for the partially integrable case is derived. Moreover, the existence of an inverse function utilized in the algorithm is proven. After that, an example is presented for evaluation of the effectiveness of the proposed method. As a result, it turns out that the proposed integrating algorithm can easily calculate independent first integrals for given partially integrable FRACs, and thus this new algorithm is expected to be applied to various research fields.
\end{abstract}

\section{Keywords}

Fully Rheonomous Affine Constraints, Geometric Representation, Rheonomous Bracket, Partial Integrability, Integrating Algorithm

\section{Introduction}

A lot of researches on nonholonomic systems have been actively done in the research fields of nonlinear control theory and robotics so far [1]-[5]. Moreover, sub-Riemannian geometry has been also studied in the research fields of differential geometry and control theory [6] [7]. In these two research fields, the common key is the existence of constraints, which is represented as differential equations. The concepts "integrability" and "nonintegrability" of such constraints are quite important since they determine various characteristics of systems. Hence, 
investigation of integrability and nonintegrability is an essential task in the first stage.

There are some classes in constraints, and the simplest class of constraints is called linear constraints: $B(q) \dot{q}=0, q \in \mathbf{R}^{n}, B \in \mathbf{R}^{(n-m) \times n}$, which has been mainly studied so far. The class of the linear constraints covers wide-ranging mechanical systems such as mobile caars, acrobatic robots, and biomimetic robots. As a wider class of constraints than the linear constraints, the author has focused and researched scleronomous affine constraints: $A(q)+B(q) \dot{q}=0, A \in \mathbf{R}^{(n-m)}$, and A-rheonomous affine constraints: $A(t, q)+B(q) \dot{q}=0$ from the viewpoints of mathematics and control theory [8]-[12]. It must be noted that in the field of analytical mechanics, the terminology "rheonomous" means "time-varying," and the opposite word of it is "scleronomous." The affine constraints can be found in physical and mechanical systems: space robots with initial angular momenta, a ball on a rotating table, a ship on a running river, and so on. These work have made it possible to treat such constraints systematically, however, we are still interested in fully rheonomous affine constraints:

$A(t, q)+B(t, q) \dot{q}=0$ as a much wider class of constraints than the scleronomous affine constraints. In [13], the author has derived a complete integrability condition for the fully rheonomous affine constraints. Moreover, in [14], an integrating algorithm to calculate first integrals of the completely integrable fully rheonomous affine constraints is developed. The word "complete integrability" means all the constraints are integrable. However, there is another case called "partial integrability", which means the situation where integrable constraints and nonintegrable ones are mixed. Researches on partially integrable fully rheonomous affine constraints have not been done.

Hence, the aim of this paper is to develop partial integrability conditions and an integrating algorithm for the fully rheonomous affine constraints. This paper is organized as follows. First, in Section 2, some preliminaries on the fully rheonomous affine constraints are shown. Next, in Section 3, necessary and sufficient conditions on partial integrability for the fully rheonomous affine constraints are derived. Then, an integrating algorithm to calculate independent first integrals for partially integrable rheonomous affine constraints is developed in Section 4. After that, Section 5 illustrates in order to verify the effectiveness and the availability of the new results.

\section{Preliminaries}

\subsection{Fully Rheonomous Affine Constraints (FRACs)}

This section presents some preliminaries on fully rheonomous affine constraints are presented. See [13] for more details. First, this subsection gives the definition of fully rheonomous affine constraints and explains their geometric representation. Denote the time variable by $t \in \mathbf{R}$ and a time interval by $I \subset \mathbf{R}$. Let $Q$ be an $n$-dimensional configuration manifold and $q=\left[\begin{array}{lll}q_{1} & \cdots & q_{n}\end{array}\right]^{\mathrm{T}} \in Q$ be a local coordinate of $Q$. Associated with $q$, we refer $\dot{q}=\left[\begin{array}{lll}\dot{q}_{1} & \cdots & \dot{q}_{n}\end{array}\right]^{\mathrm{T}} \in T_{q} Q$ as a tangent vector field. A set of $n-m(m<n)$ of differential equations in the form:

$$
A_{i}(t, q)+B_{i 1}(t, q) \dot{q}_{1}+\cdots+B_{i n}(t, q) \dot{q}_{n}=0, \quad i=1, \cdots, n-m .
$$

is called fully rheonomous affine constraints (FRACs). Note that all the coefficients $A_{i}, B_{i j}, i=1, \cdots, n-m, j=1, \cdots, n$ explicitly depend on the time variable $t$. We now rewrite (1) as

$$
A(t, q)+B(t, q) \dot{q}=0
$$

where a rheonomous affine term $A(t, q) \in \mathbf{R}^{n-m}$ is a vector-valued function whose $i$-th entry is $A_{i}(t, q)$, and $a$ rheonomous velocity coefficient matrix $B(t, q)$ is a matrix-valued function whose ij-th entry is $B_{i j}(t, q)$. In this paper, we assume the following sufficient condition on independency of the FRACs (2):

$$
\operatorname{rank} B(t, q)=n-m, \forall t \in I, \forall q \in Q .
$$

Next, a geometric representation method of the FRACs (2) is explained. From (3), we see that the $n-m$ row vectors of $B(t, q)$ in (2) are independent of each other. Hence, we consider $m$ vector fields which are independent of each other and annihilators of the $n-m$ row vectors of $B(t, q)$, and denote them by $Y_{1}(t, q), \cdots, Y_{m}(t, q)$ as time-varying vector fields on $Q$. Furthermore, we also denote a space spanned by $Y_{1}, \cdots, Y_{m}$, that is, a time-varying distribution on $Q$ by

$$
D(t, q):=\operatorname{span}\left\{Y_{1}(t, q), \cdots, Y_{m}(t, q)\right\} .
$$

Since the basial vectors of $D: Y_{1}, \cdots, Y_{m}$ are independent of each other, $D$ is a nonsingular distribution, that is, 


$$
\operatorname{dim} D(t, q)=m, \forall t \in I, \forall q \in Q
$$

holds. A curve on $Q: q: I \rightarrow Q$ is said to satisfy the FRACs (2) if for a time-varying vector field on $Q: X$ and the generalized velocity of $q: \dot{q} \in T_{q(t)} Q$,

$$
\dot{q}(t)-X(t, q(t)) \in D(t, q(t)), \forall t \in I
$$

holds. We call $X$ a rheonomous affine vector field and it satisfies the equation:

$$
A(t, q)+B(t, q) X(t, q)=0, \forall t \in I, \forall q \in Q .
$$

This definition is a natural extension of the one for the scleronomous affine constraints that do not contain the time variable explicitly [8]. Geometric representation of the FRACs is defined as follows and can allow us to analyze them geometrically and derive geometric properties.

Definition 1: The FRACs (2) are geometrically represented by a pair $(D, X)$, where $D$ is an $m$-dimensional time-varying distribution defined by (4) and $X$ is called a rheonomous affine vector and satisfies (7).

\subsection{Rheonomous Bracket}

Next, this subsection explains a new operator for the fully rheonomous affine constraints (2), called the rheonomous bracket. The rheonomous bracket is originally introduced in order to analyze the A-rheonomous affine constraints in [10]-[12], and plays important roles in derivation of a complete integrability condition and an integrating algorithm. The rheonomous bracket is fundamentally defined based on the normal Lie bracket $[\cdot, \cdot]: T M \times T M \rightarrow T M$ which is an operator for two vector fields $Z, W:$

$$
[Z, W]:=\frac{\partial W}{\partial q} Z-\frac{\partial Z}{\partial q} W
$$

The definition of the rheonomous bracket for the FRACs (2) is as follows [10]-[12].

Definition 2 [10]-[12]: For the vector fields defined on $Q$ on the geometric representation of the FRACs (2): $X, Y_{1}, \cdots, Y_{m}$, the rheonomous bracket is an operator: $\langle\cdot, \cdot\rangle: T Q \times T Q \rightarrow T Q$ that satisfies the following three properties:

(a) For a rheonomous affine vector field $X$,

$$
\langle X, X\rangle=0
$$

holds.

(b) $D_{0}$ is defined as a set of vector fields that consists of $Y_{1}, \cdots, Y_{m}$ and iterated rheonomous brackets of $X, Y_{1}, \cdots, Y_{m}$ and does not contain $X$. For a rheonomous affine vector field $X$ and a vector field $Z \in D_{0}$,

$$
\langle X, Z\rangle=\frac{\partial Z}{\partial t}+[X, Z],\langle Z, X\rangle=-\frac{\partial Z}{\partial t}+[Z, X]
$$

holds.

(c) For two vector fields $Z, W \in D_{0}$,

$$
\langle Z, Z\rangle:=0, \quad\langle Z, W\rangle:=[Z, W]
$$

holds.

For the rheonomous bracket, it is noted that the rheonomous affine vector field $X$ is perceived as special, and this yields an additional term of a time differential of a vector field as the property (b). It must be also noted that from Definition 2 the rheonomous bracket is equivalent to the normal Lie bracket for scleronomous affine constraints, that is, constraints that do not contain the time variable explicitly. The following proposition shows that the rheonomous bracket has some important characteristics in common with the normal Lie bracket [10]-[12]. 
Proposition 1: For the vector fields on the geometric representation of the FRACs (2): $X, Y_{1}, \cdots, Y_{m}$ and the set of iterated vector fields of them: $D_{0}$, the following properties (a), (b), and (c) hold.

(a) Bilinearlity:

$$
\begin{aligned}
& \langle X, a Z+b W\rangle=a\langle X, Z\rangle+b\langle X, W\rangle, \\
& \langle a Z+b W, X\rangle=a\langle Z, X\rangle+b\langle W, X\rangle, a, b \in \mathbf{R}, Z, W \in D_{0} .
\end{aligned}
$$

(b) Skew-symmetry:

$$
\langle X, Z\rangle=-\langle Z, X\rangle, Z, W \in D_{0}
$$

(c) Jacobi’s identity:

$$
\langle\langle X, Z\rangle, W\rangle+\langle\langle Z, W\rangle, X\rangle+\langle\langle W, X\rangle, Z\rangle=0, \quad Z, W \in D_{0}
$$

From Proposition 1, it can be confirmed that we only have to consider the iterated rheonomous brackets in the form:

$$
P:=\left\langle P_{k},\left\langle P_{k-1},\left\langle\cdots,\left\langle P_{2}, P_{1}\right\rangle, \cdots\right\rangle\right\rangle\right\rangle, \quad P_{1}, \cdots, P_{k} \in\left\{X, Y_{1}, \cdots, Y_{1}\right\}
$$

in checking a complete integrability condition for the FRACs, which will be shown in the next subsection. Furthermore, the Philip Hall basis [2], which is a systematic method to generate iterated Lie brackets with an order efficiently, can be also constructed for the rheonomous bracket as follows [10]-[12].

Algorithm 1: For iterated rheonomous brackets (15) of the geometric representation of the FRACs (2): $X, Y_{1}, \cdots, Y_{m}$, we define the length of $(15)$ as $l(P)=k$, that is, the number of vector fields in the iterated rheonomous bracket. In addition, the symbol $\prec$ means the magnitude relation for two iterated rheonomous brackets. Then, the Philip Hall basis $H$ for the rheonomous bracket can be constructed by the next rules.

(a) $X, Y_{1}, \cdots, Y_{m}$ are the first $m+1$ elements of $H$ and $X \prec Y_{1} \prec \cdots \prec Y_{m}$.

(b) If $l\left(P^{1}\right)<P\left(P^{2}\right)$, then $P^{1} \prec P^{2}$.

(c) $\left\langle P^{1}, P^{2}\right\rangle \in H$ if and only if $P^{1}, P^{2} \in H$ and $P^{1} \prec P^{2}$, either $P^{1}=X$ or $P^{1}=Y_{i}(i=1, \cdots, m)$ holds or $P^{1}=\left\langle P^{3}, P^{4}\right\rangle$ with $P^{3}, P^{4} \in H$ and $P^{3} \preceq P^{4}$.

\section{Partial Integtability Conditions for FRACs}

In this section, a partial integrability condition for the FRACs (2) is derived. In the simplest terms, the partially integrable case means that some constraints are integrable and the others are nonintegrable. If the $n-k$ FRACs in (2) are integrable, that is, there exist $n-k$ independent first integrals of (2), then they are said to be $k$-th order partially integrable. Now, we define a smallest and involutive time-varying distribution $C_{0}(t, q)$ which contains $Y_{1}, \cdots, Y_{m}$ and iterated rheonomous brackets of them, and satisfies $\langle X, W\rangle \in C_{0}, \forall W \in C_{0}$, that is, $C_{0}$ is spanned by all the rheonomous brackets of $X, Y_{1}, \cdots, Y_{m}$ with the exception of $X$. Then, necessary and sufficient conditions on partilly integrability for the FRACs (2) are given as the next theorem.

Theorem 1: For the fully rheonomous affine constraints defined on an $n$-dimensional manifold $Q$ (2) and a time interval $I \subset \mathbf{R}$, the following statements (a) and (b) are equivalent to each other. If they hold, the fully rheonomous affine constraints (2) are said to be $k$-th order partially integrable $(m<k<n)$.

(a) There exist $n-k$ independent first integrals of the fully rheonomous affine constraints (2).

(b) For a smallest and involutive time-varying distribution $C_{0}(t, q)$,

$$
\operatorname{dim} C_{0}(t, q)=k, \forall t \in I, \forall q \in Q
$$

holds. 
(Proof) Let us consider the $(n+1)$-dimensional product space $\bar{Q}:=\mathbf{R} \times Q$, where $\mathbf{R}$ is the space of the time variable $t$, and the local coordinate of $\bar{Q}$ is given by $\bar{q}:=\left[\begin{array}{ll}t & q^{\mathrm{T}}\end{array}\right]^{\mathrm{T}}$. On $\bar{Q}$, the FRACs (2) can be represented by Pfaffian equations of $n-m$ differential forms:

$$
A(t, q) \mathrm{d} t+B(t, q) \mathrm{d} q=0 .
$$

Since the rheonomous affine vector field $X$ of geometric representation satisfies (7), $m+1$ vector fields on $\bar{Q}$ which annihilate (17) are given by

$$
\bar{X}(t, q):=\frac{\partial}{\partial t} \oplus X(t, q), \bar{Y}_{i}(t, q):=0 \oplus Y_{i}(t, q), \quad i=1, \cdots, m .
$$

We now define an involutive distribution $\bar{C}$ defined on $\bar{Q}$ which contains $\bar{X}, \bar{Y}_{1}, \cdots, \bar{Y}_{m}$ and iterated Lie brackets for $\bar{X}, \bar{Y}_{1}, \cdots, \bar{Y}_{m}$. Therefore, by Frobenius' theorem [1] [2] [15] [16] and the results [13] [14], It can be confirmed that a necessary and sufficient condition on $k$-th order partial integrability for (17) is given by

$$
\operatorname{dim} \bar{C}(\bar{q})=k+1, \forall \bar{q} \in \bar{Q} .
$$

Calculating the iterated Lie brackets for $\bar{X}, \bar{Y}_{1}, \cdots, \bar{Y}_{m}$, then we obtain

$$
\begin{aligned}
& {\left[\bar{X}(t, q), \bar{Y}_{i}(t, q)\right]=0 \oplus\left\langle X(t, q), Y_{i}(t, q)\right\rangle,} \\
& {\left[\bar{X}(t, q),\left[\bar{X}(t, q), \bar{Y}_{i}(t, q)\right]\right]=0 \oplus\left\langle X(t, q),\left\langle X(t, q), Y_{i}(t, q)\right\rangle\right\rangle,} \\
& \vdots \\
& {\left[\bar{Y}_{j}(t, q), \bar{Y}_{i}(t, q)\right]=0 \oplus\left\langle Y_{j}(t, q), Y_{i}(t, q)\right\rangle,} \\
& {\left[\bar{Y}_{l}(t, q),\left[\bar{Y}_{j}(t, q), \bar{Y}_{i}(t, q)\right]\right]=0 \oplus\left\langle Y_{l}(t, q),\left\langle Y_{j}(t, q), Y_{i}(t, q)\right\rangle\right\rangle,}
\end{aligned}
$$

We can easily see that $\bar{X}$ is independent of $\bar{Y}_{i}, \cdots, \bar{Y}_{m}$ and the iterated Lie brackets (20). Hence, we can change the necessary and sufficient condition (19) into the condition such that $\bar{Y}_{1}, \cdots, \bar{Y}_{m}$ and the iterated Lie brackets which consist of $\bar{X}, \bar{Y}_{1}, \cdots, \bar{Y}_{m}$ span an $k$-dimensional space. From the calculations (18) and (20), we only have to consider $Y_{1}, \cdots, Y_{m}$ on $Q$ instead of $\bar{Y}_{1}, \cdots, \bar{Y}_{m}$ on $\bar{Q}$, and iterated rheonomous brackets for $X, Y_{1}, \cdots, Y_{m}$ on $Q$ instead of Lie brackets for $\bar{X}, \bar{Y}_{1}, \cdots, \bar{Y}_{m}$ on $\bar{Q}$. Consequently, a necessary and sufficient condition on $k$-th order partially integrability for the FRACs (2) is that $Y_{1}, \cdots, Y_{m}$ and the iterated rheonomous brackets for $X, Y_{1}, \cdots, Y_{m}$ span an $k$-dimensional space, that is, (16) holds.

From Theorem 1, it turns out that the partial integrability condition for the FRACs (2) is quite simple and is a natural extension of the case of the complete integrability case [13] [14]. Moreover, we can see that the rheonomous bracket plays a significant role in the condition (16).

\section{Integrating Algorithm for Partially Integrable FRACs}

\subsection{Integrating Algorithm}

The result in Section 3 shows that if the FRACs (2) are $k$-th order partially integrable, there exist $n-k$ independent first integrals of them. For reduction of the dimension of a given configuration manifold subject to partially integrable FRACs, we need the explicit forms of independent first integrals of them. The author has developed algorithms to calculate independent first integrals of them in the case of complete integrability [14]. However, the algorithm cannot be utilized for the case of partial integrability. Hence, in this subsection, an integrating algorithm for partially integrable FRACs (2) is constructed.

Assume that the FRACs (2) satisfy Theorem 1, that is, they are $k$-th order partially integrable. Then, we can find $n-k$ vector fields $Y_{m+1}, \cdots, Y_{k}$ that satisfy

$$
C_{0}=\operatorname{span}\left\{Y_{1}, \cdots, Y_{m}, Y_{m+1}, \cdots, Y_{k}\right\} .
$$

In addition, set linearly independent vector fields $Y_{k+1}, \cdots, Y_{n}$ for $X, Y_{1}, \cdots, Y_{k}$, that is

$$
\operatorname{span}\left\{Y_{1}, \cdots, Y_{k}, Y_{k+1}, \cdots, Y_{n}\right\}=\mathbf{R}^{n},
$$


holds. Then, let us denote flows (1-parameter local transformation groups) of $X$ and $Y_{i}$ by $\phi_{t}^{X}$ and $\varphi_{\tau_{i}}^{Y_{i}}$ with parameters $t$ and $\tau_{i}$, respectively. We set an initial point at the initial time $t=0$ as $q^{0} \in Q$. We also consider $m+1$ vector fields defined on the expanded configuration manifold $\bar{Q}=\mathbf{R} \times Q$ (18), and then their flows on $Q$ are obtained as

$$
\bar{\phi}_{t}^{\bar{X}}:=\left[\begin{array}{c}
t \\
\varphi_{t}^{X}
\end{array}\right], \bar{\varphi}_{\tau_{i}}^{\bar{Y}_{i}}:=\left[\begin{array}{c}
0 \\
\varphi_{\tau_{i}}^{Y_{i}}
\end{array}\right], i=1, \cdots, m .
$$

Calculating the composite mapping of $(n+1)$ flows (23) yields

$$
\bar{\Phi}:=\bar{\phi}_{t}^{\bar{X}} \circ \bar{\varphi}_{\tau_{1}}^{\overline{Y_{1}}} \circ \cdots \circ \bar{\varphi}_{\tau_{n}}^{\bar{Y}_{n}}=\left[\begin{array}{c}
t \\
\phi_{t}^{X} \circ \varphi_{\tau_{1}}^{Y_{1}} \circ \cdots \circ \varphi_{\tau_{n}}^{Y_{n}}
\end{array}\right]=\left[\begin{array}{c}
t \\
\Phi_{t}
\end{array}\right],
$$

where

$$
\Phi_{t}:=\phi_{t}^{X} \circ \varphi_{\tau_{1}}^{Y_{1}} \circ \cdots \circ \varphi_{\tau_{n}}^{Y_{n}}
$$

is the composite mapping of $n+1$ flows $\phi_{t}^{X}, \varphi_{\tau_{i}}^{Y_{i}}$. From (24), we can see that the projection of $\bar{\Phi}$ onto $Q$ is equivalent to $\Phi_{t}$. Hence, by applying the idea of the integrating algorithm for scleronomous linear constraints defined on $Q$ [1] [2] to $\mathbf{R} \times Q$ and considering projection of it onto $Q$, Consequently, we have the following algorithm to calculate $n-k$ independent first integrals of partially integrable FRACs.

Algorithm 2: For the case where the A-rheonomous affine constraints (2) are $k(m<k<n)$-th order partially integrable, we can obtain $n-k$ independent first integrals of them by the following procedure.

Step 1: Set $m+1$ vector fields $X, Y_{1}, \cdots, Y_{m}$ of geometric representation for the FRACs (2).

Step 2: By calculating iterated rheonomous brackets of $X, Y_{1}, \cdots, Y_{m}$, derive $k-m$ linearly independent vector fields $Y_{m+1}, \cdots, Y_{k}$ that satisfy

$$
C_{0}=\operatorname{span}\left\{Y_{1}, \cdots, Y_{m}, Y_{m+1}, \cdots, Y_{k}\right\} .
$$

Step 3: For $X, Y_{1}, \cdots, Y_{k}$, derive linearly independent vector fields $Y_{k+1}, \cdots, Y_{n}$ that satisfy

$$
\operatorname{span}\left\{Y_{1}, \cdots, Y_{k}, Y_{k+1}, \cdots, Y_{n}\right\}=\mathbf{R}^{n} .
$$

Step 4: Calculate flows (1-parameter local transformation groups) of $X, Y_{i}$ with parameters $t, \tau_{i}$, respectively: $\phi_{t}^{X}, \varphi_{\tau_{i}}^{Y_{i}}$.

Step 5: Combine $n+1$ flows calculated in Step 4 as

$$
\Phi_{t}:=\phi_{t}^{X} \circ \varphi_{\tau_{1}}^{Y_{1}} \circ \cdots \circ \varphi_{\tau_{n}}^{Y_{n}} .
$$

Step 6: Set $q=\Phi_{t}(\tau)$ and calculate the inverse function $\tau=\Phi_{t}^{-1}(q)$. Then, the last $n-k$ components of $\Phi_{t}^{-1}$ are first integrals of $k$-th order partially integrable FRACs (2).

\subsection{Theoretical Analysis of Integrating Algorithm}

Next, this subsection gives theoretical analysis on the integrating algorithm for partially integrable FRACs (Algorithm 2). In Algorithm 2, we have to derive the inverse function of the combined mapping: $\Phi_{t}^{-1}$ in order to calculate independent first integrals. However, the existence of the inverse function is not guaranteed. In general, it is quite difficult to calculate an inverse function of a given function. For Algorithm 2, the next proposition guarantees the existence of the inverse mapping $\Phi_{t}^{-1}$.

Proposition 2 Assume that the FRACs (2) are $k$-th order partially integrable. Then, there exists an time interval $I \subset \mathbf{R}$ and $\Phi_{t}: \mathbf{R}^{n} \rightarrow Q$ is a diffeomorphism at any time $t \in I$. That is to say, there exists its inverse mapping $\Phi_{t}^{-1}$. 
(Proof) Set $\bar{\tau}:=\left[\begin{array}{ll}t & \tau^{\mathrm{T}}\end{array}\right]^{\mathrm{T}}$. Calculating the partial differential of (24) with the chain rule of differential calculation, we have

$$
\begin{aligned}
\frac{\partial \bar{\Phi}}{\partial t} & =\frac{\partial}{\partial t}\left\{\bar{\phi}_{t}^{\bar{X}} \circ \bar{\varphi}_{\tau_{1}}^{\bar{Y}_{1}} \circ \cdots \circ \bar{\varphi}_{\tau_{n}}^{\bar{Y}_{n}}\right\}=\bar{X}\left(\bar{\phi}_{t}^{\bar{X}} \circ \bar{\varphi}_{\tau_{1}}^{\bar{Y}_{1}} \circ \cdots \circ \bar{\varphi}_{\tau_{n}}^{\bar{Y}_{n}}\right), \\
\frac{\partial \bar{\Phi}}{\partial \tau_{i}} & =\frac{\partial \bar{\phi}_{t}^{\bar{X}}}{\partial \bar{\tau}} \frac{\partial \bar{\varphi}_{\tau_{1}}^{\bar{Y}_{1}}}{\partial \bar{\tau}} \cdots \frac{\partial \bar{\varphi}_{\tau_{i-1}}^{\bar{Y}_{i-1}}}{\partial \bar{\tau}} \frac{\partial}{\partial \tau_{i}}\left(\bar{\varphi}_{\tau_{i}}^{\bar{y}_{i}} \circ \cdots \circ \bar{\varphi}_{\tau_{n}}^{\bar{Y}_{n}}\right) \\
& =\frac{\partial \bar{\phi}_{t}^{\bar{X}}}{\partial \bar{\tau}} \frac{\partial \bar{\varphi}_{\tau_{1}}^{\bar{Y}_{1}}}{\partial \bar{\tau}} \cdots \frac{\partial \bar{\varphi}_{\tau_{i-1}}^{\bar{Y}_{i-1}}}{\partial \bar{\tau}} \bar{Y}_{i}\left(\bar{\varphi}_{\tau_{i}}^{\bar{Y}_{i}} \circ \cdots \circ \bar{\varphi}_{\tau_{n}}^{\bar{Y}_{n}}\right) .
\end{aligned}
$$

Substituting $\bar{\tau}=\bar{\tau}^{0}=\left[\begin{array}{llll}t^{0} & 0 & \cdots & 0\end{array}\right]^{\mathrm{T}}$ into (29), we obtain

$$
\left.\frac{\partial \bar{\Phi}}{\partial t}\right|_{\bar{\tau}=\bar{\tau}^{0}}=\bar{X}\left(q^{0}\right),\left.\quad \frac{\partial \bar{\Phi}}{\partial \tau_{i}}\right|_{\bar{\tau}=\bar{\tau}^{0}}=\bar{Y}_{i}\left(q^{0}\right),
$$

that is to say,

$$
\left.\frac{\partial \bar{\Phi}}{\partial \bar{\tau}}\right|_{\bar{\tau}=\bar{\tau}^{0}}=\left[\begin{array}{llll}
\bar{X}\left(q^{0}\right) & \bar{Y}_{1}\left(q^{0}\right) & \cdots & \bar{Y}_{n}\left(q^{0}\right)
\end{array}\right]
$$

Since $\bar{X}, \bar{Y}_{1}, \cdots, \bar{Y}_{n}$ are linearly independent of each other,

$$
\left.\operatorname{rank} \frac{\partial \bar{\Phi}}{\partial \bar{\tau}}\right|_{\bar{\tau}=\bar{\tau}^{0}}=n+1
$$

holds. Therefore, it turns out that $\bar{\Phi}(\bar{\tau})$ is a diffeomorphism by the implicit function theorem [15] [16]. Since the projection of $\bar{\Phi}(\bar{\tau})$ onto $Q$ is equivalent to $\Phi_{t}(\tau), \Phi_{t}(\tau)$ is also a diffeomorphism. Consequently, the proposition is proven.

From Proposition 2, we can see that similar to the case of complete integrability [14], the existence of the inverse mapping $\Phi_{t}^{-1}$ is also guaranteed. For an example of calculation of independent first integrals for the partially integrable FRACs, see the next section.

\section{Example}

Finally, this section deals with an example to evaluate the new results. Let us consider a 3-dimensional configuration manifold:

$$
Q=\left\{q=\left[\begin{array}{lll}
q_{1} & q_{2} & q_{3}
\end{array}\right]^{\mathrm{T}} \in \mathbf{R}^{3}\right\}
$$

with $n=3$, and a fully rheonomous affine constraints on $Q$ :

$$
\underbrace{\left[\begin{array}{c}
2 t \\
q_{3}
\end{array}\right]}_{A(t, q)}+\underbrace{\left[\begin{array}{ccc}
1 & 0 & 0 \\
0 & t & 0
\end{array}\right]}_{B(t, q)}\left[\begin{array}{c}
\dot{q}_{1} \\
\dot{q}_{2} \\
\dot{q}_{3}
\end{array}\right]=0
$$

with $m=1$. We here consider a time interval $I:=(0, \infty)$. Then, it turns out that Assumption 1 holds for (34). One geometric representation for (34) can be obtained as follows:

$$
X=\left[\begin{array}{c}
-2 t \\
-\frac{q_{3}}{t} \\
0
\end{array}\right], Y=\left[\begin{array}{l}
0 \\
0 \\
1
\end{array}\right] .
$$

Calculating an iterated rheonomous bracket for $X$ and $Y$ above, we obtain 


$$
\begin{aligned}
& \langle X, Y\rangle=\frac{\partial Y}{\partial t}+[X, Y]=\left[\begin{array}{l}
0 \\
\frac{1}{t} \\
0
\end{array}\right], \\
& \langle X,\langle X, Y\rangle\rangle=\frac{\partial\langle X, Y\rangle}{\partial t}+[X,\langle X, Y\rangle]=\left[\begin{array}{c}
0 \\
-\frac{1}{t^{2}} \\
0
\end{array}\right] \\
& \langle Y,\langle X, Y\rangle\rangle=0 .
\end{aligned}
$$

Since $\langle X, Y\rangle \|\langle X,\langle X, Y\rangle\rangle$ holds, we have

$$
C_{0}=\operatorname{span}\{Y,\langle X, Y\rangle\}
$$

and then it can be confirmed that

$$
\operatorname{dim} C_{0}(t, q)=2, \forall t \in I, \forall q \in Q
$$

holds. From Theorem 1, it turns out that the FRACs (34) are 2nd order partially integrable $(k=2)$, that is, there exists one independent first integrals of (34).

Next, we shall calculate the first integrals of (34) according to Algorithm 1. Set $Y_{1}:=Y, Y_{2}:=\langle X, Y\rangle$ and a new vector field that satisfy (26) as

$$
Y_{3}:=\left[\begin{array}{l}
1 \\
0 \\
0
\end{array}\right] .
$$

For the vector fields $X, Y_{1}, Y_{2}, Y_{3}$, we calculate their flows as

$$
\begin{gathered}
\phi_{t}^{X}=\left[\begin{array}{c}
-t^{2}+\left(t^{0}\right)^{2}+q_{1}^{0} \\
q_{2}^{0}+q_{3}^{0} \log \frac{t^{0}}{t} \\
q_{3}^{0}
\end{array}\right], \varphi_{\tau_{1}}^{Y_{1}}=\left[\begin{array}{c}
q_{1}^{0} \\
q_{2}^{0} \\
\tau_{1}+q_{3}^{0}
\end{array}\right], \\
\varphi_{\tau_{2}}^{Y_{2}}=\left[\begin{array}{c}
q_{1}^{0} \\
q_{2}^{0}+\log \frac{\tau_{2}}{\tau_{2}^{0}} \\
q_{3}^{0}
\end{array}\right], \quad \varphi_{\tau_{3}}^{Y_{3}}=\left[\begin{array}{c}
\tau_{3}+q_{1}^{0} \\
q_{2}^{0} \\
q_{3}^{0}
\end{array}\right],
\end{gathered}
$$

where $q^{0}=\left[\begin{array}{lll}q_{1}^{0} & q_{2}^{0} & q_{3}^{0}\end{array}\right]^{\mathrm{T}} \in Q$ is the initial point at the initial time $t=t^{0}$. Combining the flows (40) like (28), we have

$$
\Phi_{t}(\tau)=\phi_{t}^{X} \circ \varphi_{\tau_{1}}^{Y_{1}} \circ \phi_{\tau_{2}}^{Y_{2}} \circ \phi_{\tau_{3}}^{Y_{3}}=\left[\begin{array}{c}
\tau_{3}-t^{2}+\left(t^{0}\right)^{2}+q_{1}^{0} \\
\left(\tau_{1}+q_{3}^{0}\right) \log \frac{t^{0}}{t}+\log \frac{\tau_{2}}{\tau_{2}^{0}}+q_{2}^{0} \\
\tau_{3}+q_{3}^{0}
\end{array}\right] .
$$

By solving the equation $q=\Phi_{t}(\tau)$, we calculate the inverse mapping of (41) as 


$$
\Phi_{t}^{-1}(q)=\left[\begin{array}{c}
q_{3}-q_{3}^{0} \\
\mathrm{e}^{q_{2}-q_{2}^{0}} \tau_{2}^{0}\left(\frac{t}{t^{0}}\right)^{q_{3}} \\
t^{2}-\left(t^{0}\right)^{2}+q_{1}-q_{1}^{0}
\end{array}\right] .
$$

Consequently, we can obtain one independent first integrals of (34) as the last component of (43):

$$
h_{1}(t, q)=t^{2}-\left(t^{0}\right)^{2}+q_{1}-q_{1}^{0} .
$$

\section{Conclusions}

This paper has studied necessary and sufficient conditions for the fully rheonomous affine constraints (FRACs) in the case of partial integrability, and develops an integrating algorithm in order to calculate independent first integrals. It turns out that the new proposed integrating algorithm is expected to be a powerful mathematical tool and can be utilized in various research fields.

Future work includes applications of the new results to various systems, extensions of sub-Riemannian geometry, and analysis of more general classes of constraints.

\section{References}

[1] Isidori, A. (1995) Nonlinear Control Systems. 3rd Edition, Springer-Verlag, London. http://dx.doi.org/10.1007/978-1-84628-615-5

[2] Sastry, S.S. (1999) Nonlinear Systems. Springer-Verlag, New York. http://dx.doi.org/10.1007/978-1-4757-3108-8

[3] Cortés, J. (2002) Geometric, Control and Numerical Aspects of Nonholonomic Systems. Springer-Verlag, Berlin Heidelberg. http://dx.doi.org/10.1007/b84020

[4] Bloch, A.M. (2003) Nonholonomic Mechanics and Control. Springer-Verlag, New York. http://dx.doi.org/10.1007/b97376

[5] Bullo, F. and Rewis, A.D. (2004) Geometric Control of Mechanical Systems. Springer Science+business Media, Inc.

[6] Montgomery, R. (2002) A Tour of Subriemannian Geometries, Their Geodesics and Applications. American Mathematical Society.

[7] Calin, O. and Change, D.C. (2009) Sub-Riemannian Geometry: General Theory and Examples. Cambridge University Press, Cambridge. http://dx.doi.org/10.1017/CBO9781139195966

[8] Kai, T. and Kimura, H. (2006) Theoretical Analysis of Affine Constraints on a Configuration Manifold—Part I: Integrability and Nonintegrability Conditions for Affine Constraints and Foliation Structures of a Configuration Manifold. Transactions of the Society of Instrument and Control Engineers, 42, 212-221.

[9] Kai, T. (2011) Integrating Algorithms for Integrable Affine Constraints. IEICE Transactions on Fundamentals of Electronics, Communications and Computer Sciences, E94-A, 464-467.

[10] Kai, T. (2012) Mathematical Modelling and Theoretical Analysis of Nonholonomic Kinematic Systems with a Class of Rheonomous Affine Constraints. Applied Mathematical Modelling, 36, 3189-3200. http://dx.doi.org/10.1016/j.apm.2011.10.015

[11] Kai, T. (2012) Theoretical Analysis for a Class of Rheonomous Affine Constraints on Configuration Manifolds—Part I: Fundamental Properties and Integrability/Nonintegrability Conditions. Mathematical Problems in Engineering, 2012, Article ID: 543098.

[12] Kai, T. (2012) Theoretical Analysis for a Class of Rheonomous Affine Constraints on Configuration Manifolds—Part II: Foliation Structures and Integrating Algorithms. Mathematical Problems in Engineering, 2012, Article ID: 345942.

[13] Kai, T. (2013) On Integrability of Fully Rheonomous Affine Constraints. International Journal of Modern Nonlinear Theory and Application, 2, 130-134. http://dx.doi.org/10.4236/ijmnta.2013.22016

[14] Kai, T. (2013) An Integrating Algorithm and Theoretical Analysis for Fully Rheonomous Affine Constraints: Completely Integrable Case. Applied Mathematics, 4, 1720-1725. http://dx.doi.org/10.4236/am.2013.412235

[15] Nomizu, S. and Kobayashi, K. (1996) Foundations of Differential Geometry Volume I. Wiley-Interscience.

[16] Nomizu, S. and Kobayashi, K. (1996) Foundations of Differential Geometry Volume II. Wiley-Interscience. 